\section{Gefährliche Tage für Asthmatikerinnen}

\author{
Seit Jahrzehnten gibt es die Hypothese, dass mit Beginn der \\ Menstruation das Risiko eines schweren Asthmaanfalles steigt. \\ Evidenzbasierte Daten dazu aus einem großen Kollektiv fehlten \\ allerdings bisher.
}

S panische Forscher sammelten im Rahmen einer HochrisikoasthmaMultizenterstudie über einen Zeitraum von 2 Jahren insgesamt 128 Fälle von Frauen mit beinahe letalen Asthmaanfällen. Die Daten von 44 Frauen im reproduktiven Alter wurden dann in eine genauere Analyse eingeschlossen und nach demographischen Charakteristika, Verlauf des chronischen Asthmas in den vergangenen 12 Monaten, klinischen Charakteristika sowie Auslösern der fast letalen Asthmaanfälle weiter aufgeschlüsselt. Anschließend wurde das Kollektiv in zwei Gruppen unterteilt und das Risiko eines fast letalen Asthmaanfalls entweder am ersten Tag der Menstruation oder an jedem weite-

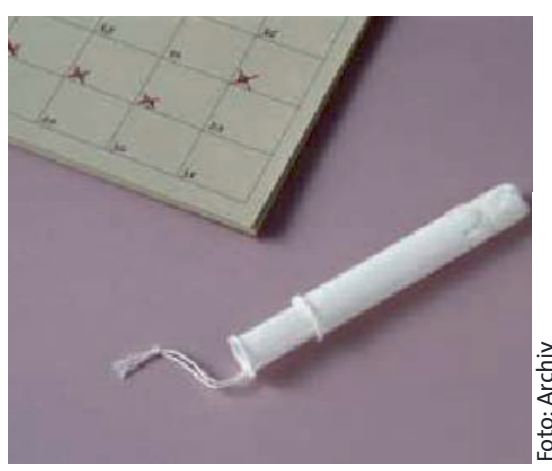

Der Beginn der Menstruation kann Auslöser eines schweren oder sogar fast letalen Asthmaanfalles sein.

ren Tag der Blutung getrennt ermittelt.
Es stellte sich heraus, dass es am ersten Tag der Blutung signifikant häufiger zu schweren Asthmaanfällen kam als an jedem weiteren Tag $(p=0,022)$. Gleichzeitig benötigten die Patientinnen, die zu Beginn der Menstruation einen Asthmaanfall erlitten, bereits 7 Tage vor dem schweren Anfall wesentlich mehr Medikamente $(p=0,003)$. Zudem hatten diese Patientinnen in der Vorgeschichte wesentlich häufiger schwere oder fast letale Asthmaanfälle.

Fazit: Diese Daten bringen Gewissheit, dass es eine Beziehung zwischen Menstruation und Asthmaanfällen gibt. Betroffene Frauen sollten auf ihr spezielles Risiko hingewiesen und vor allem in der Schwangerschaft optimal therapiert werden. Interessant wären weitere Untersuchungen zu den noch nicht bekannten physiologischen und möglicherweise auch psychologischen Mechanismen. of

Martinez-Moragon E et al. Near-fatal asthma related to menstruation. J Allergy Clin Immunol 2004; 113: 242-4

\title{
Fatale Fitness - Asthmatod beim Sport
}

\section{Eine Korrelation zwischen der Sterblichkeit von Asthmatikern und körperlicher Betätigung wurde in der Literatur immer wieder beschrieben. Allerdings gab es bisher keinerlei Hinweise, dass Sport an sich für Asthmatiker ein tödliches Risiko sein kann.}

\footnotetext{
Corscher des Temple Sports Asthma Research Centers in Philadelphia, USA, sammelten die Daten von 263 Personen, die während oder kurz nach sportlicher Betätigung verstorben waren. Daraus wurden 61 Fälle isoliert, bei denen retrospektiv als Todesursache eindeutig ein Asthmaanfall festgestellt werden konnte.

Von den Verstorbenen waren 81\% jünger als 21 Jahre, die Hälfte verstarb im Alter zwischen 13 und 17 Jahren. $61 \%$ der Untersuchten waren männlich, das Verhältnis Weiße zu Schwarze lag bei zwei zu eins. Obwohl bei 91\% der Betroffenen eine asthmatische Vorgeschichte bestand, wandten lediglich 5\% (drei Personen) regelmäßig Medi-
}

kamente an. Interessant ist auch, dass Wettkampfsportler mit $57 \%$ ein geringfügig höheres Risiko für einen plötzlichen Tod hatten als Freizeitsportler. Die häufigsten letalen Asthmaanfälle traten in dieser Untersuchung übrigens bei Basketballspielern auf, Schwimmer waren dagegen sehr selten betroffen.

Fazit: Körperliche Belastung kann offenbar ein bisher nicht bekanntes letales Risiko für Asthmatiker darstellen. Dennoch sollte man Asthmatikern den Sport sicher nicht verbieten. Voraussetzung für eine risikolose körperliche Betätigung ist dabei die optimale Therapie. Verantwortliche wie Trainer und Leh-
Tödliche Asthmaanfälle beim Sport nach ausgeübter Sportart

\begin{tabular}{lc|} 
Sportart & Anzahl \\
Basketball & 13 \\
Laufsport & 7 \\
Gymnastik & 6 \\
Football & 5 \\
Bolzspiele & 5 \\
Baseball & 3 \\
Fußball & 3 \\
Schwimmen & 2 \\
Cheerleading & 2 \\
Sonstige & 15 \\
Summe & 61 \\
\hline
\end{tabular}

rer sollten spezielle Schulungen erhalten, um Risiken erkennen und lebensrettende Erstmaßnahmen durchführen zu können.

\section{oj}

Becker JM et al: Asthma deaths during sports: report of a 7-year experience. J Allergy Clin Immunol 2004; 113: 264-7 